\title{
AN UPDATE ON X-RAY EMISSION FROM STARS
}

\author{
R. ROSNER \\ Enrico Fermi Institute and Dept. of Astronomy and Astrophysics \\ The University of Chicago \\ 5640 South Ellis Avenue \\ Chicago, IL 60637
}

\begin{abstract}
With the closing of the Einstein and EXOSAT eras, the characteristics of stellar xray emission are now fairly well understood. In this very brief review, I will focus on two specific topics central to the physics underlying stellar activity: the rotation-activity connection and the "decay" of activity at the low mass end of the main sequence.
\end{abstract}

\section{Introduction}

Given the brevity of space for this report, I have chosen to focus on two topics which I think well-illustrate both the breadth of results now available for stellar activity studies and the depth of analysis now possible: the nature of the activity-rotation connection, and the nature of activity at the low-mass end of the main sequence. Both topics were recognized early on to be central to our understanding of how stellar activity comes about; and the recent results which I shall discuss provide significant information on this central problem of stellar activity.

Of necessity, a substantial number of interesting topics will not be discussed here: the novel high time resolution radio observations of stellar activity reported by $\mathrm{J}$. Bookbinder, G. Dulk, and collaborators; the new correlation studies of x-ray luminosity and UV flux reported by K. Schrijver and collaborators; the novel theoretical modeling of hot and cool "loops" by S. Antiochos and G. Noci; and the remarkable "stellar"-like flare observed by J. Schmitt, F.R. Harnden, and H. Fink by using Einstein data of florescent emission from the solar flare-illuminated terrestrial atmosphere. This omission is regrettable, but unavoidable.

\section{Is Rotation the Determinant of Stellar X-ray Emission?}

In a recent preprint, Fleming, Gioia, and Maccacaro (1988) have used the Extended Medium Sensitivity Survey (EMSS) from Einstein to construct an X-ray-selected sample of 128 F-M dwarf stars, and have combined this sample with optical observations (including spectroscopy) to establish the relations between spectral type, rotation rate, $\mathrm{x}$ ray luminosity, etc. among these sample stars. 
The key result is shown in their Figure 1, which seems to indicate that unlike the classical result of Pallavicini et al. (1981), stellar x-ray luminosity $L_{x}$ for this sample seerns to scale like $\left(v_{e} \sin i\right)^{1}$, rather than $\left(v_{e} \sin i\right)^{2}$, where $v_{e}$ is the equatorial stellar rotation rate and $i$ is the inclination angle of the stellar rotation axis to the line-of-sight. The question of exactly how $L_{x}$ scales with the rotation rate $\Omega$ has of course been a rather hotly debated topic since the early results of Pallavicini et al. and Walter (1982); and it is therefore crucial to understand the present result, which emerges from the first x-rayselected sample constructed to date for this purpose.

To begin with, we must note that since an x-ray-selected sample of the kind examined here by its very nature preferentially picks out the $x$-ray-bright sources, one way of reconciling the apparently conflicting results is if the intrisically brighter sources (e.g., the ones in the present sample) are simply showing the effect of stellar activity "saturation"; that is, it may be that at the upper end of the stellar luminosity function, the dependence of activity on rotation rate is weaker than it is for less-active stars (cf. Vilhu and Walter 1987). Can we determine whether this simple interpretation is correct?

To answer this question, Fleming et al. considered the correlations between $L_{x}, \Omega$, and stellar radius $R$; and found that (1) $L_{x}$ is uncorrelated with $\Omega \sin i$, while (2) the x-ray luminosity instead scales with $R^{2}$. These latter results seem to deepen the mystery: it appears that for this well-determined sample of late-type dwarf stars, it is the stellar radius (or surface area) which sets the $\mathrm{x}$-ray emission level, not the stellar rotation rate. Is there a simple explanation for the discrepancy between this result and earlier studies?

The following argument seems to me to resolve the difficulty. Since the sample we are considering is x-ray-selected, and indeed represents the upper tail of the $L_{x}$ distribution, one might expect that the stars in this sample have mean surface fluxes which are comparable to those in solar active regions; this is in fact borne out by comparing $L_{x}$ of stars in this sample with the expected $L_{x}$ if these stars were fully covered by solar-like active regions (cf. Vaiana and Rosner 1978). In that case, we expect that the x-ray luminosity of stars in this sample can be "predicted" simply by determining the stellar surface area for each star, and multiplying this area by the mean solar active region x-ray flux; that is, we would expect a strong correlation between $L_{x}$ and the square of the stellar radius for stars in this sample. This is exactly what is found by Fleming et al.

However, recall that $v_{\varepsilon}=\Omega R$, and that Fleming et al. found $L_{x}$ to be uncorrelated with $\Omega \sin i$ for this sample; the implication is then immediate that $L_{x} \approx v_{e}{ }^{2}$, contrary to what is in fact found - Fleming et al. find instead a linear dependence of $L_{x}$ on $v_{\varepsilon}$. Is there then an internal inconsistency in the data analysis? I believe not. Careful reading of Fleming et al.'s analysis shows that the power law fit connecting $L_{x}$ to $v_{e} \sin i$ is obtained by simply assuming that the upper limits on rotation rates $v_{e} \sin i<10 \mathrm{~km} / \mathrm{s}$ can be regarded as "true detections", with fractionally-large errors, and applying a leastsquares fit to all of the data. The effect is to overweight (with respect to the upper bounds) the few "detections" at large rotation rates and relatively low luminosities, and thus to underestimate the exponent in the power law. This can be avoided by instead applying the by-now standard detection-and-bounds correlation analysis (cf. Schmitt et al. 1985). Indeed, if one uses the data presented in Table 1 of Fleming et al. to compute the power law index, one finds that within the statistical errors of the data, the power law index connecting $v_{e}$ to $L_{x}$ for this sample cannot be distinguished from 2! This is remarkable: the exponent is 2 despite the fact that $L_{z}$ for this sample is demonstrably uncorrelated with stellar rotation. I suspect that if the $L_{x} \approx v_{t}{ }^{2}$ relation had been found first, then there would have been little reason to probe further, since this result would have confirmed the "well-known" result that activity and rotation are correlated in 
precisely this manner; it is to Fleming et al.'s credit that they did in fact probe further, and now provide the first good evidence for saturation of stellar coronal activity.

A rather different perspective on the activity-rotation problem is provided by the recent completion of the Einstein survey of late-type giants and supergiants (Maggio et al. 1988). The total sample encompassed 380 stars, and was used in the first instance to resolve the problem of locating the "dividing line" separating coronally-active from inactive evolved stars in the H-R diagram. Having confirmed earlier work placing this boundary at $\approx$ spectral type $K$, Maggio et al. proceeded to consider the $\mathrm{x}$-ray luminosityrotation rate correlation for this stellar sample; it is of some considerable surprise that the sought-for correlation is remarkable weak.

The reasons for this surprise are of course the fact that for late-type dwarf stars, x-ray luminosity and rotation are well-correlated; and that there is a rather commonlyaccepted and intuitively-appealing picture of how this correlation might come about. However, the data for giants serve to remind us that in some sense the data for dwarf stars are too "neat". That is, the common explanation for the activity-rotation connection is based on the idea that in standard $\alpha-\omega$ dynamo theory, the rate of magnetic flux production depends on the rate of differential rotation, and hence (for fixed convection zone depth, for example) should scale with the rotation rate. What is commonly forgotten is this latter caveat: namely, in order for this correlation to be evident, it must be the case that the depth of the region over which the differential rotation field acts either does not vary with stellar type, or varies randomly with stellar type. Thus, the x-ray data from evolved stars tell us quite directly that, given that we are wedded to standard $\alpha-\omega$ dynamo theory, the depth over which the $\omega$-dynamo must act within the convection zone of these stars must be correlated with stellar type. This would seem to be a rather stringent constraint for dynamos for evolved stars; and is confirmed by Maggio et al.'s success in correlating $L_{x}$ with the Rossby number (at least for F and early $\mathrm{G}$ giants).

\section{The "Decay" of Stellar Activity at the Low-mass End of the Main Sequence}

The possibility that the nature of stellar activity may change in some dramatic way for very low-mass stars has been the focus of detailed studies for a number of years, and plays an important role in subjects ranging from the galactic contribution to the diffuse soft x-ray background and stellar dynamo theory to the genesis of the "period gap" in cataclysmic variables (cf. Rosner, Golub and Vaiana 1985). Very recently, the Einstein Extended Medium Sensitivity Survey (EMSS), already alluded to above, has been used by Fleming, Liebert, Gioia, and Maccacaro (1988) to attack this problem as well.

Table 1. Number of predicted and observed stars as a function of detection threshold

\begin{tabular}{lccc}
\hline & Volume $\left[\mathrm{pc}^{3}\right]$ & Predicted ${ }^{[3]}$ \# $[\mathrm{Sp}>\mathrm{M} 5]$ & Observed \# $[\mathrm{Sp}>\mathrm{M} 5]$ \\
\hline $\log <\mathrm{L}_{\mathrm{x}}>[1]=28.73$ & 3,870 & 91 & 0 \\
$\log \left\langle\mathrm{L}_{\mathrm{x}}>[2]=27.98\right.$ & 265 & 6 & 0 \\
\hline
\end{tabular}

[1] Mean $L_{z}$ for young disk population stars (Bookbinder 1985), dM only.

[2] Mean $L_{x}$ for old disk population stars (Bookbinder 1985), dM only.

[3] Using stellar mass function of Reid (1987).

Fleming et al. obtain two basic results from their study of the EMSS x-ray selected sample (consisting of some $31 \mathrm{dM}$ stars, all optically identified). First, they show that 
the sample as a whole has a tendency for $\left\langle L_{2} / L_{b o l}\right\rangle$ to be roughly constant; second, they pointed out that no dM stars later than M5 were either detected or identified. The above table (extracted from Fleming et al.) summarizes the second result quantitatively, and shows quite explicitly that the absence of $\mathrm{dM}$ stars later than M5 is not likely to be a statistical fluke. Since this sample is x-ray-selected, it is clearly the most explicit demonstration of an effect first noticed in the dM star analysis of Bookbinder (1985), namely that there simply are no x-ray-bright stars later than M5 (there are certainly $x$ ray-emitting stars later than M5, but all of these stars detected to date show very modest (solar-like or less) x-ray emission levels.

The fundamental reason for this deficit of $x$-ray-bright stars remains a mystery. Rosner and Vaiana (1980) some time ago pointed out that for stars later than roughly M5, the presumptive change in stellar structure as a result of convection dominating energy transport everywhere within the star ought to be reflected in the efficiency with which such stars produce magnetic fields, and hence in how active they were; this would be especially the case if the dominant dynamo process for main sequence stars later than M5 were a "shell" dynamo located at the interface between the outer convection zone and the radiative core. This expectation is apparently borne out by the x-ray data; but unfortunately, we are no closer to understanding exactly in what way this change in stellar structure has brought about a decrease in stellar magnetic field production efficiency. That is, magnetic field production has certainly not ceased for the very low mass stars (since we still observe coronal emission), so that it cannot be simply a matter of "shutting down" field production. Instead, one presumes that the basic dynamo process has been modified; this remains a problem for the future.

\section{References}

Bookbinder, J. (1985) PhD Thesis, Harvard University.

Fleming, T.A., Gioia, I.M., and Maccacaro, T. (1988) 'The Relation Between X-ray Emission and Rotation in Late-type Stars from the Perspective of X-ray Selection', Astrophys. J., in press.

Fleming, T.A., Liebert, J., Gioia, I.M., and Maccacaro, T. (1988) 'M Dwarfs From the Einstein Extended Medium Sensitivity Survey', Astrophys. J., in press.

Maggio, A., Vaiana, G.S., Haisch, B., Stern, R.A., Bookbinder, J., Harnden, Jr., F.R., and Rosner, R. (1988) The Einstein Observatory Magnitude-limited $X$-ray Survey of Late-type Giant and Supergiant Stars', Astrophys. J., in press.

Pallavicini, R., et al. (1981) 'Relations among Stellar X-ray Emission Observed from Einstein, Stellar Rotation and Bolometric Luminosity', Astrophys. J., 248, 279.

Reid, I.N. (1987) M.N.R.A.S., 225, 873.

Rosner, R., Golub., L., and Vaiana, G.S. (1985) 'On Stellar X-ray Emission', Ann. Rev. Astron. Ap., 23, 413-52.

Rosner, R. and Vaiana, G.S. (1980) 'Stellar Coronae from Einstein: Observations and Theory', in R. Giacconi and G. Setti (eds.), X-ray Astronomy, D. Reidel Publ., Dordrecht, pp. 129-51.

Schmitt, J.H.M.M., et al. (1985) Astrophys. J., 290, 307.

Walter, F.M. (1982) Astrophys. J., 253, 745.

Vaiana, G.S. and Rosner, R. (1978) 'Recent Advances in Coronal Physics', Ann. Rev. Astron. Ap., 16, 393-428.

Vilhu, O. and Walter, F.M. (1987) Astrophys. J., 321, 958. 